\title{
Structure of Human Dicer Determined by High Throughput Single Particle Electron Microscopy
}

\author{
Pick-Wei Lau, *\# Ian MacRae,* Clinton S. Potter,\# \& Bridget Carragher\# \\ * Molecular Biology and \#Cell Biology Departments, The Scripps Research Institute, \\ 10550 North Torrey Pines Rd., La Jolla, CA 92037
}

Dicer is a specialized ribonuclease that initiates RNA interference (RNAi) by cleaving double-stranded RNA (dsRNA) into small RNA fragments about 22 nucleotides long [1]. The product of dicer is then loaded into Argonaute whereby one of the RNA strands is kept for target recognition, rendering gene-silencing effect.

The human dicer is a large $220 \mathrm{kDa}$ multi-domain protein. The only high resolution structural information of dicer is derived from the crystal structure of a much smaller homolog from Giardia intestinalis [2]. The additional domains in the human homolog are thought to play various functional roles. We set off to obtain structural insights into how machinery is put together by electron microscopy (EM).

The molecular weight and asymmetric nature of human Dicer sets itself as a challenging candidate for single particle EM. Reconstructing a small molecule requires a massive dataset, which necessitates automated data collection using Leginon [3,4] and streamlined data processing with Appion [5]. We first obtain an initial model through random conical tilt (RCT) reconstruction. Using this initial model, we refine against a large dataset comprising of more than 120,000 particles. From this, we reconstruct the threedimensional structure of human Dicer bound to the protein TRBP at approximately $20 \AA$ resolution.

Our analysis reveals that the Dicer-TRBP complex is an L-shaped molecule with a long edge of $150 \AA$ and a $100 \AA$ extension on one end. A surface trench runs the length of the long edge of the molecule, defining a putative dsRNA-binding site. Docking the crystal structure of Giardia Dicer, which represents the nuclease core of human Dicer, into the EM map suggests two possible overall molecular architectures for human Dicer.

The result of this work is a strong testimony for the necessity of automation. As more biological molecules become more challenging, automated data collection delivers the possibility of massive sample screening and data collection. With automation, this study culminates with insights into the structure of Dicer proteins found in multicellular organisms and provide a conceptual framework for understanding the initiation of RNAi [6]. 


\section{References}

[1] E. Bernstein, et al., Nature (2001) 409.

[2] I.J. Macrae, et al., Science (2006) 311.

[3] B. Carragher, et al., J Struct Biol (2000) 132.

[4] C. Suloway, et al., J Struct Biol (2005) 151.

[5] G.C. Lander, et al., J Struct Biol (2009) 166.

[6] We are grateful to Kristi E. Kass for technical support during sample preparation. Electron microscopic imaging and reconstruction were conducted at the National Resource for Automated Molecular Microscopy, which is supported by the National Institutes of Health $(\mathrm{NIH})$ through the $\mathrm{P} 41$ program of the National Center for Research Resources (RR17573). This work was also supported by the NIH grant R01 GM086701 to I.J.M. P.W.L is a predoctoral fellow of the American Heart Association. I.J.M. is a Pew Scholar in the Biomedical Sciences.

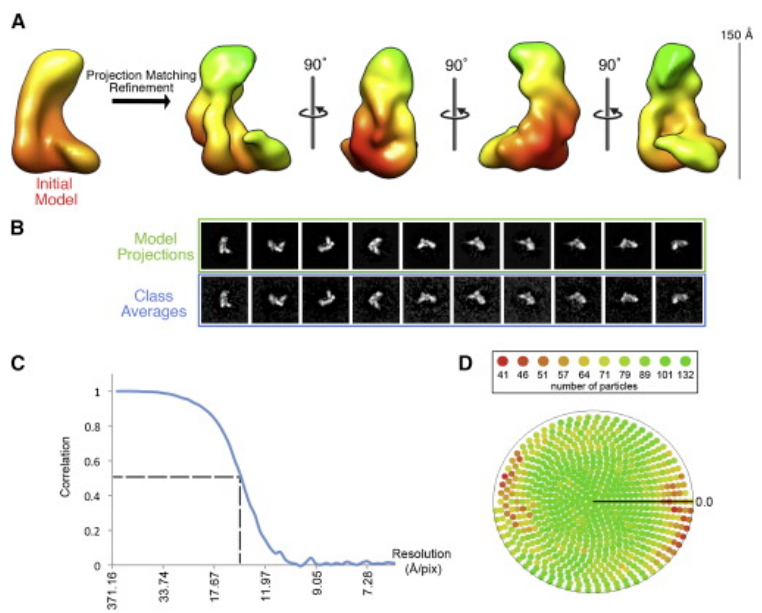

Figure 1. Iterative Refinement of the Dicer-TRBP Initial Model. Using the refined initial model, projection matching iterative refinement was carried out against a 130,000particle data set.(A) Four views of the resulting Dicer-TRBP model.(B) The model projections in comparison with the class averages at the final iteration of the refinement protocol.(C) The FSC curve of the final iteration of the refinement. At a 0.5 cutoff, the resolution is determined to be approximately $15 \AA$.(D) The particle distribution across all possible Euler angles at the final refinement iteration.
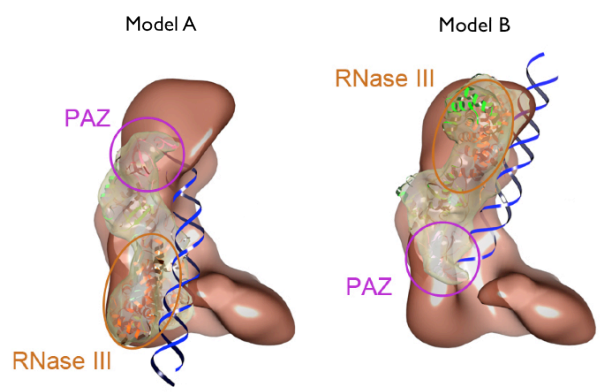

Figure 2: Docking of Giardia dicer nuclease core into EM density. Two possible orientations of Giardia dicer crystal structure is being fitted into the EM density of Human dicer. 